\title{
FUNCTIONAL MRI ACTIVATION SIGNAL DETECTION USING THE PERIODICITY TRANSFORM
}

\author{
Ajay V. Deshmukh', Vivek Shivhare, \\ Randhir Singh Parihar, Vikram M. Gadre \\ 'deshmukh@ee.iitb.ac.in, ajavd1967@yahoo.com \\ Indian Institute of Technology, Bombay \\ Electrical Engineering Department \\ Powai, Mumbai, 400 076, India
}

\author{
Deepak P. Patkar, Smruti Shah \\ and Sona Pungavkar \\ New MRI Centre \\ Nanavati Hospital, Mumbai \\ Vile Parle (W), Mumbai, 400056 India
}

\begin{abstract}
A key challenge in Functional Magnetic Resonance Imaging (fMRI) is the detection of activation areas in the brain. We introduce a new method of fMRI signal detection, using an approach termed the Periodicity Transform. The technique is based on temporal data analysis. A search for periodicity is carried out in the fMRI lime series data. The method is applicable to block design experiments. In the block paradigm, the stimulus period is known and it is possible to use this information for searching periodicities in the time series data. We present the results for the periodicity detection in the time serics of the simulated phantom as well as clinical fMRI data from the finger tapping experiment. No assumptions have been made about the amplitude and frequency of the activation signal. The algorithm extracts arbitrary harmonics at the periodicity defined by the stimulus function.
\end{abstract}

\section{INTRODUCTION}

Functional MRI studies are used to understand the brain functions. fMRI linds a number of applications in neurosciences and surgical planning. In an fMRI experiment, the haemodynamic response of the brain is measured. The subject performs either a sensory or motor task, while recording the data. $\mathrm{MRI}$ is a non-invasive technique and the experiment can be repeatedly carried out. The technique of fMRI is based on the blood oxygenation level dependent contrast (BOLD) [1,2]. This blood oxygenation level change gives rise to a temporal signal that changes $T_{2}^{*} . T_{2}^{*}$ is a combined measure of local magnetic field inhomogeneity and intrinsic spin-spin relaxation mechanisms. Normally an Echo Planner imaging (EPI) scquence is used for image acquisition and a time series of images is recorded. A block paradigm experiment is generally carried out with $O N$ (activation) and OFF (baseline or rest condition) blocks of certain duration. The signal resulting from an $\mathrm{fMRI}$ experiment is very small in magnitude. Approximately 2 to 4 percent of baseline change

The authors thank Dr. Nagraj G. Huilgol, Society for Cancer Research and Communication India and New MRI Center, Nanavati Hospital Mumbai for encouraging this research. occurs in the signal. The signal is also contaminated with a significant amount of noise resulting in poor signal to noise ratios (SNR). Noise in fMRI consists of physiological noise and scanner induced noise. We propose a method of fMRI signal analysis, based on the Periodicity transform approach [3] for a block paradigm fMRI experiment. A priori information about the stimulus is available in all the block paradigm experiments. The activationbaseline pattern is periodic; therefore we assume periodic nature of the output signal having the same fundamental frequency as that of the stimulus [4].

\section{THE PERIODICITY TRANSFORM}

Finding periodicities in a given set of data is one of the needs. in signal and image processing. The periodicity transform [3] decomposes a sequence into a sum of periodic sequences. Projecting the input sequence onto a set of periodic subspaces $P_{p}$ does this. The Periodicity transform decomposition is in terms of periodic sequences; therefore it is linear in period. The basis vectors of the periodicity transform are not specified a priori, but the transform itself tinds its own best set of basis elements. Let us define the periodic subspace $P_{p}$ as the set of all $p$-periodic sequences and $P$ as the set of all periodic sequences. Further, $P_{p} \subset P$. We call a sequence of real numbers $x(k)$ as $p$-periodic if there exists an integer $p$ such that $x(k+p)=x(k)$ for all integers $k$. If the length of the sequence $x(k)$ is $N$, then $N$ is assumed to be a single period. This is simply stated as $x_{N} \in P_{N}$. Now, one is interested in finding out the periodicities in $x_{N}(k)$ which are smaller than $N$. This is done in the periodicity transform, by projecting $x_{N}(k)$ on to the subspaces $P_{p}$ for $p<N$. If $x_{N}$ is close to any periodic subspace $P_{p}$, then there is a $p$-periodic element $x_{p}$ close to the original sequence $x$. This $x_{p}$ is used to decompose $x$. 


\subsection{Projecting the Signal onto the Periodic Subspaces}

Projecting the signal onto the periodic subspaces does the Periodicity analysis. The signal $\mathrm{x}$ is assumed to be periodic or $x \in P_{p}$ for some $p$. Let $x_{p}^{*}$ be the orthogonal projection of $x$ onto $P_{p}$. Then, the optimal $x_{p}^{*}$ can be expressed as a linear combination of the periodic basis elements $\delta_{p}^{s}$.

$$
x_{p}^{*}=\alpha_{0} \delta_{p}^{0}+\alpha_{1} \delta_{p}^{1}+\ldots+\alpha_{p-1} \delta_{p}^{p-1}
$$

Where,

$$
\delta_{p}^{s}(j)=\left\{\begin{array}{cc}
1 & \text { if }(\mathrm{j}-\mathrm{s}) \bmod \mathrm{p}=0 \\
0 & \text { Otherwise }
\end{array} .\right.
$$

The coefficients $\alpha_{s}$ are given by

$$
\alpha_{s}=\frac{1}{N / p} \sum_{n=0}^{N / p-1} x(s+n p)
$$

Where $N / p$ must be an integer. The Periodicity transform approach is different from frequency-based methods in the sense that a periodic signal is also periodic with integer multiples of the periods and the order in which various periodicities are extracied is important. The projection of signal $x$ onto $P_{p}$ is given by

$$
\pi\left(x, P_{p}\right)=\sum_{n=0}^{p-1} \alpha_{n} \delta_{p}^{n}
$$

Where $\pi$ is the projection operator.

\subsection{Periodicity Transform Algorithms}

Periodicity analysis decomposes a signal into basic periodic components. Unlike Fourier analysis the periodic components are not necessarily sine and cosine waves. Different algorithms have been described by Sethares and Staley [3] in their work on the Periodicity Transforms. Those are Small to Large, Best-Frequency, Best-Correlation, Mbest, and Mhestgama. Small to large finds periodicities regularly. The Best frequency algorithm sometimes gets stuck, if the frequency does not correspond to some integer periodicity. The Best-correlation algorithm works properly when the data is spiky. The Mbest algorithm finds multiples of basic periodicities also. Mbestgama is more reliable in the presence of noise [5]. In the fMRI time series analysis our interest is to find the periodicity at the period of the stimulus, therefore we prefer the M-Best algorithm. Best frequency and Best correlation algorithms are not considered because this is not a frequency or correlation based analysis. Small to large algorithm is not used since we look for only one periodicity at a time. The Mbest algorithm extracts and maintains the list of the $M$ best periodicities and the corresponding basis elements. The value of $M$ is an input parameter to the algorithm. These $M$ periodicities remove most of the energy, in the sense of the norm measure, from the sequence. The projections onto the larger subspaces contain the smaller periodicities and are again decomposed. If the smaller periodicities remove more energy than those in the current list of extracted periodic elements, then the newly extracted periodic element replaces the old. Figure 1 shows the functional block schematic of the Mbest algorithm. Just to see

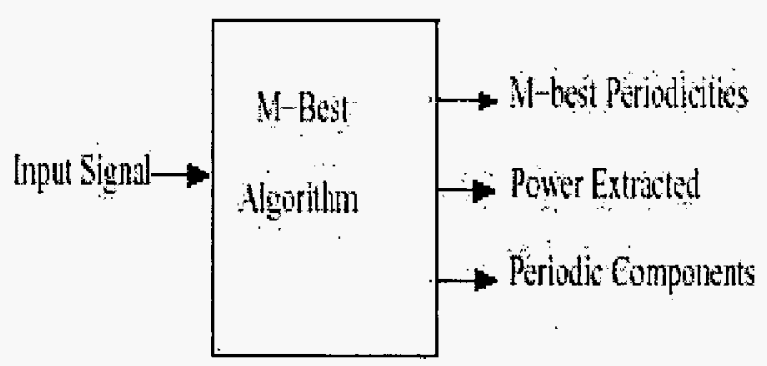

Fig. 1. Mbest algorithm

the operation of the periodicity transform using the Mbest algorithm, consider true signal as shown in Figure 2. A noisy signal was formed as shown in Figure 3, where the signal itself comprises a pure sine wave of periodicity 20 and another periodic signal of sinusoidal nature with a frequency corresponding to a periodicity of 13.33 . The periodicity of 13.33 of a sinusoidal waveform does not belong to any of the harmonics of the true signal. In Figure 4 the signal, which is

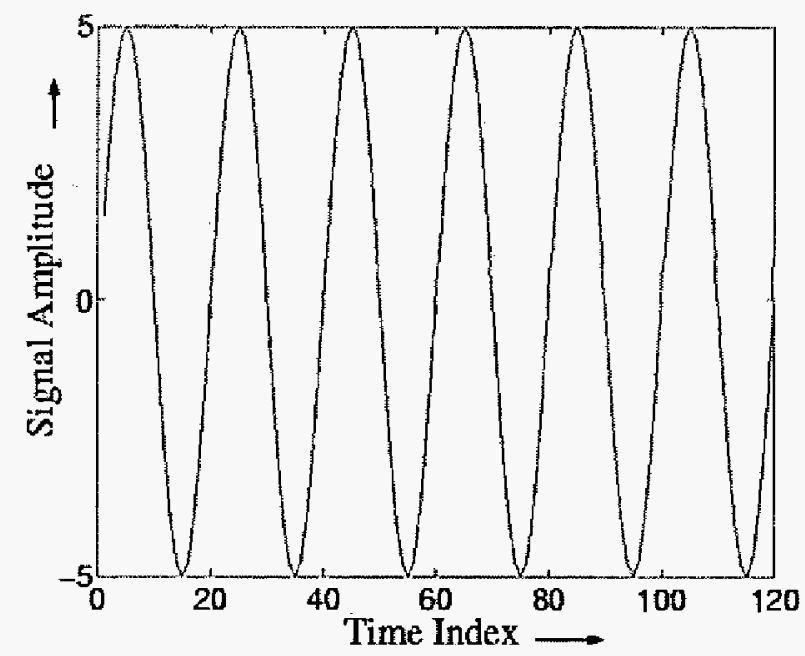

Fig. 2. Original sinusoidal signal (periodicity $=20$ )

extracted using the Mbest algorithm, is shown. It must be noted here that our interest in extracting the periodicity does not include the exact waveshape of the true signal, rather it is the periodic component at the desired periodicity may be with some distortion.

\section{THE PERIODICITY TRANSFORM AND FMRI DATA ANALYSIS}

Functional MRI data analysis often involves some kind of signal model or haemodynamic response model. In this paper a Periodic nature of fMRI signal is assumed, which is 
applicable to block design fMRI experiments only. The method is elaborated in the following subsection.

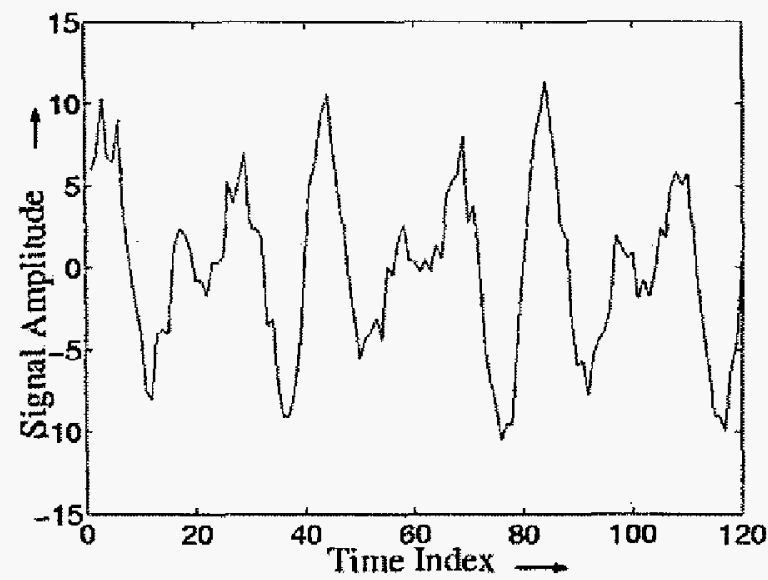

Fig. 3. Signal and noise with another periodic component, which is not a harmonic of the true signal

\subsection{The Periodicity Transform Approach to fMRI Data Analysis}

The Periodicity Transform can be used to analyze the data where it is believed to have some periodic component of interest. In fMRI time series data there are periodicities due to cardiac pulsations, and the activation signal as well. The period of the stimulus is of utmost interest for the detection of activated pixels. The fMRI signal can be modeled as a periodic component at the period of the stimulus with an arbitrary number of harmonics. Further the phase lag of the time series will have spatial dependence. We already have assumed that there is some periodic component in the fMRI time series data, which corresponds to the activated pixels. The inactivated pixels are only characterized by the noisy time series. However, the activated time series is noisy and its periodicity may not be visible directly.

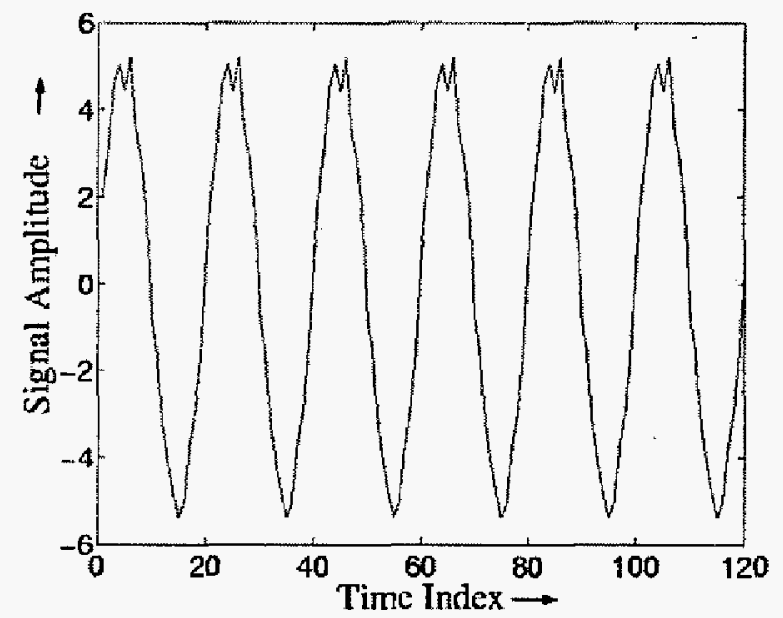

Fig. 4. Extracted signal from noisy data using the Mbest algorithm
The Periodicity transform can detect the periodicities in the noisy waveform [3]. The Periodicity transform does the best to enhance the power of the periodic component, irrespective of the precise waveform in one period. Therefore, a threshold on extracted power at the period of stimulus can be used to sort out the activated pixels from the fMRI dataset. We consider the input stimulus $u(t)$ consisting of $M_{O N}$ blocks and $M_{\text {OFF }}$ blocks. It means that there are $M=\left(M_{O N}+M_{O F F}\right) / 2$ cycles of stimulus. The baseline and activation images are acquired alternately during the whole experiment. In each cycle there are $N_{O N}+N_{O F F}$ frames. In our experiment $N_{O N}=N_{O F F}$ which results in a square wave stimulus. Let the image sequence recorded during the fMRI experiment be $I\left(k_{1}, k_{2}, l\right)$. Where $k_{1}$ and $k_{2}$ are spatial dimensions and $l$ is the discrete time index. The time series data corresponding to a pixel $Z\left(k_{\mathrm{L}}, k_{2}\right)$ is $T S\left(k_{1}, k_{2}, l\right)$. The pixel located at $\left(k_{1}, k_{2}\right)$ can be declared to be activated, if there is a periodicity at the period given by stimulus, and with sufficient signal power. Such a group of activated pixels form a spatial activation pattem. The fMRI time series can be considered to have both periodic and nonperiodic components. Further, out of all periodic components the signal is assumed to be only at periodicity equal to that of stimulus $u(t)$. Thus all the periodic components at periods other than stimulus period as well as those, which are nonperiodic, form the noise in the fMRI time series data. Thus the fMRI time series $T S(\mathbf{k}, l)$ can be represented by,

$$
T S(\mathbf{k}, l)=\pi\left(T S(\mathbf{k}, l), P_{T}\right)+\sum_{i} \pi\left(T S(\mathbf{k}, l,), P_{p_{l}}\right)+v(l)
$$

Where $\pi\left(T S, P_{T}\right)$ is the projection of $T S$ onto the $T$ periodic basis elements. The projection operation is given by equation (4). $p_{i}$ are periodicities other than the stimulus period $T$ and $v$ is the non periodic noisy component. The first term represents the projection of the time series onto the $T$ periodic basis functions of the Periodicity Transform. The second term consists of all the periodic components present in the time series data, at periodicities other than $T$. There can be an arbitrary number of periodic components in the second term. The third term is the non periodic noisy component. Our method of Periodicity transform differs from all the other similar methods, in that it explicitly seeks the term $\pi\left(T S(\mathbf{k}, I), P_{T}\right)$ in the equation (5) above, which captures a specific periodic component without constraint on waveshape. This is what is important from an fMRI perspective. To the best of our knowledge, no other method directly seeks periodicity and compacts its presence into a single term as in equation (5) without specific reference to the periodic waveshape. 


\section{THE IMRI DATA}

\subsection{Simulated Data}

For a first verification of the algorithm, data was created through software simulation. This is a time series of length 120. A Gaussian random noise was added to the time series. The pure simulated fMRI activation signal is assumed to be a sine wave of period 20 plus its second harmonic. This corresponds to a signal resulting from a stimulus with $6 \mathrm{ON}$ and 6 OFF blocks. The simulated signal is given by

$$
x(k)=\sin (2 . \pi \cdot f \cdot k / 120)+\sin (2 \pi \cdot 2 \cdot f \cdot k / 120)+\eta(k)
$$

Where $\eta(k)$ is the gaussian random noise and $f=6$ is the number of ON/OFF blocks. Figure 5 shows the original sinusoidal signal with two harmonics (at the top), the middle panel shows the noisy time series, with the SNR of $0.9350 \mathrm{~dB}$. The lower panel shows the extracted signal using the Mbest algorithm. The reason for using two harmonics is that the periodic fMRI signal may have more than one sinusoidal frequency and it is the repetitive pattern at the stimulus period, which is of interest.

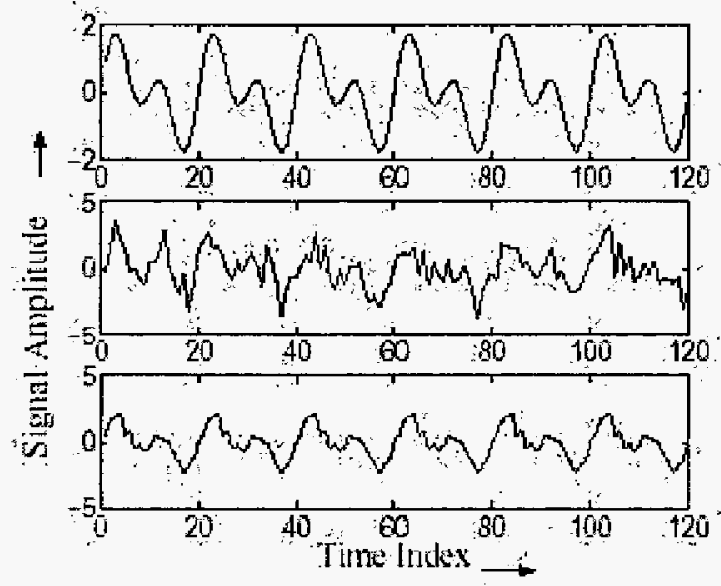

Fig. 5. Simulated signal without any noise is shown on the top. The middle panel shows the noisy time series with SNR of $0.9350 \mathrm{~dB}$. The extracted signal at the periodicity of 20 , using the periodicity transform (Mbest algorithm) is shown at the bottom

\subsection{Real fMRI Data}

The fMRI experiment was carried out on the GE signa 1.5-T scanner. The subject was asked to perform the finger-tapping task with both hands and the EPI images were recorded. The data set consists of four slices each of size 64x64 pixels, cutting through the motor cortex. For each of the slices a time series of 128 images was recorded. This generated a data set of dimensions $4 \times 64 \times 64 \times 128$. Gradient echo sequence was used with time interval between successive scans (TR) of 3000 msec, and echo time of $60 \mathrm{msec}$. To compensate for the delay in the haemodynamic response we neglected the first images at the beginning of each block in the ON-OFF pattem and finally it reduced to a $4 \times 64 \times 64 \times 122$ matrix of data. Our analysis needs the length of time series equal to some multiple of the stimulus period, in this case 20 . Therefore in the actual analysis, we considered the first 120 images out of 122 again resulting into a data set of dimensions $4 \times 64 \times 64 \times 120$. The data set was recorded at the New MRI center, Nanavati Hospital, Vile-Parle, Mumbai, India.

\section{IMRI DATA PROCESSING}

The input to our algorithm is the time series belonging to the intracranial pixels. We calculated the average of all the images in the time series belonging to the slice. The histogram of the average image, as shown in Figure 6 , is of weakly bimodal nature and the valley region was used to decide on a threshold. Thus pixels belonging to the brain area were identified.

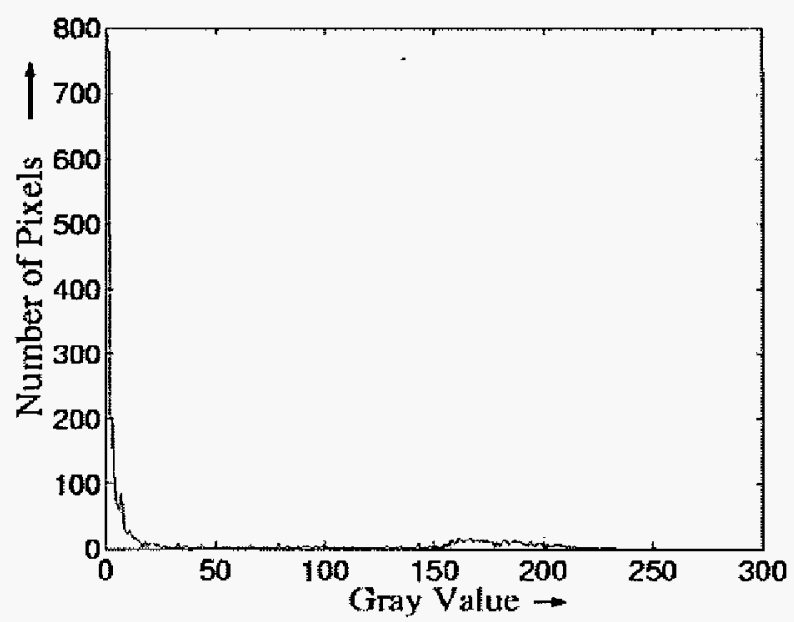

Fig. 6. Histogram of Averaged Image

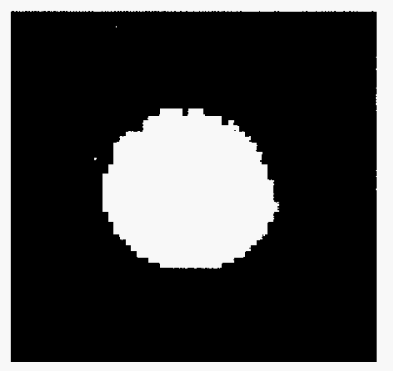

Fig. 7. Mask from Averaged Image

The valley point was selected such that none of the intracranial pixels are discarded. The hard tissues which have intensities lesser than the valley threshold point are eliminated this way. Figure 7 show the mask obtained using this procedure. Next, the periodicity transform was applied to the time series data belonging to the intracranial region. The Mbest algorithm calculates the periodicity within the time series. The output parameters are the normalised norm after 
each periodicity, the number of periodicities detected along with the periodicity values. Those pixel time series, which exhibit a strong periodic component at the stimulus period, are marked as activated. If the periodicity extracted matched with 20 then the power of the extracted signal was tested.

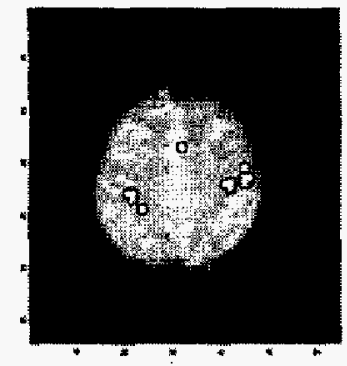

(a) Slice 1

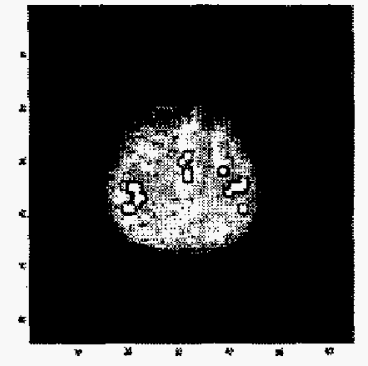

(b) Slice 2
Fig. 8. Activation Pattern

The threshold was entered in an interactive manner manually in the range between 0 to 1 . The code was written in MATLAB.

\section{RESULTS AND DISCUSSIONS}

We have processed the clinical fMRI data using the Mbest algorithm. Figure 8 shows the results obtained by processing slice 1 and slice 2 of the clinical data obtained from the finger tapping experiment. The activated pixels are marked with white color. A threshold of 0.82 in terms of signal power was applied manually. Figure 9 shows one of the activated time series. The periodic nature of the extracted signal can be observed. From Figures 8 (a) and (b) the activated motor areas can be seen clearly. The method of periodicity extraction was found to be very simple and efficient. The algorithm is highly time-efficient it takes only a few minutes for the total computation. As a comparison the popular SPM-99 software takes about an hour to do similar processing and involves spatial smoothing. It must be noted that any prior filtering is highly undesirable in fMRI data processing. Our method uses the data set without any filtering. This is possible in the Periodicity Transform approach because of the fact that the Mbest Periodicity Transform algorithm works well even in low SNR conditions. The method of Periodicity transform is deterministic and not based upon any statistical model. Even though the periodicity at the stimulus period is important, however there are no restrictions on the exact frequencies or amplitudes extracted by the algorithm. This itself makes the procedure of Periodicity analysis a robust one. The method is less sensitive to motion artifacts, because the Periodicity analysis itself is not much affected by any transient appearing in the time series. The longer length of time series as compared with the time intervals during which motion occurs can justify this. This is further supported by the fact that the exact shape of the periodic component is not very crucial. Next, the waveshape distortion of the periodic component due to small motion does not affect the algorithm significantly.

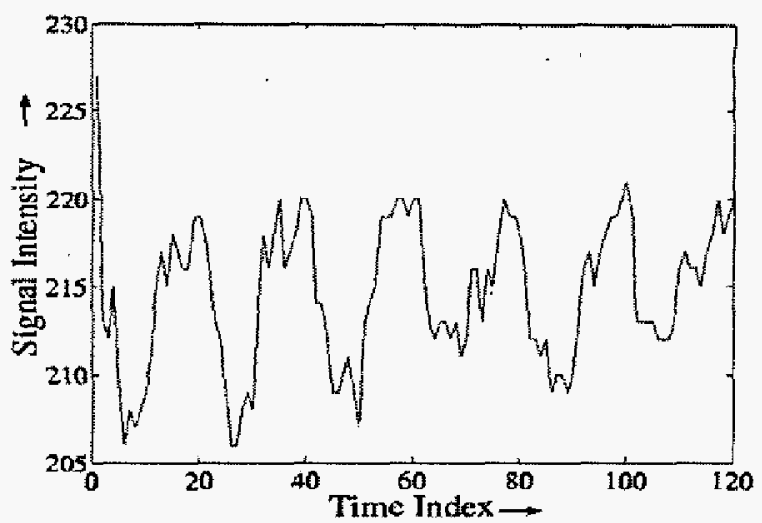

Fig. 9. Activated time series

\section{CONCLUSION}

A novel method for detecting the activated areas in brain from fMRI signals has been developed, based upon the periodicity transform. The algorithm was verified on the simulated phantom data and then applied to real fMRI data. The signa] detection is quite reliable even in presence of noise. The algorithm works well even for the noise levels found in typical IMRI data. Except for the manual thresholding part, periodicities are detected automatically. There is no loss of spatial or temporal information.

\section{REFERENCES}

[1] S. Ogawa, T. M. Lee, A. S. Nayak and P. Glynn, Oxygenation-sensitive contrast in magnetic resonance image of rodent brain at high fields, Magnetic Resonance in Medicine 14 (1990) 68-78.

[2] O. Josephs, R. Turner and K. Friston, Event-relared fMRI, Human Brain Mapping 5 (1997) 243-248.

[3] W. A. Sethares, T. W. Staley, Periodicity transforms, IEEE Trans. on Signal Processing 47 (1 1) (1999), 2953-2964

[4] K. K. Babak A. Ardekani, Jeff Kershaw, I. Kanno, Activation detection in functional MRI using Subspace Modelling and Maximum Likelihood Estimation, IEEE Trans. Med. Imag. 18 (2) (1999), 101-114.

[5] MATLAB programs for the calculation of the periodicity transforms[online]available http://eceserv0.ece.wisc.edu/sethares. 